\title{
Screening for and combining serum intestinal barrier-related biomarkers to predict the disease severity of AECOPD
}

\author{
Jiuheng Yin ${ }^{1 \#}$, Shuai Wang ${ }^{1 \#}$, Yuan Qiu ${ }^{1}$, Enlai Jiang ${ }^{1}$, Guangsheng Du ${ }^{1}$, Wensheng Wang ${ }^{1}$, Pengyuan Xu ${ }^{2}$, \\ Hua Yang ${ }^{1}$, Mingdong $\mathrm{Hu}^{1}$, Weidong Xiao ${ }^{1}$ \\ ${ }^{1}$ Department of General Surgery, Xinqiao Hospital, Army Military Medical University, Chongqing, China; ${ }^{2}$ Department of Gastrointestinal Surgery, \\ Second Affiliated Hospital of Kunming Medical University, Kunming, China \\ Contributions: (I) Conception and design: M Hu, W Xiao; (II) Administrative support: H Yang; (III) Provision of study materials or patients: G Du, \\ W Wang; (IV) Collection and assembly of data: S Wang, Y Qiu, J Yin, E Jiang; (V) Data analysis and interpretation: J Yin, S Wang, P Xu; (VI) \\ Manuscript writing: All authors; (VII) Final approval of manuscript: All authors. \\ \#These authors contributed equally to this work. \\ Correspondence to: Weidong Xiao, MD, PhD. Department of General Surgery, Xinqiao Hospital, 183 Xinqiao Main Street, Shapingba, Chongqing \\ 400037, China. Email: weidong.xiao@126.com; Mingdong Hu, MD, PhD. The Intensive Care Unit of the Department of Respiratory Medicine, \\ Xinqiao Hospital, 183 Xinqiao Main Street, Shapingba, Chongqing 400037, China. Email: mingdonghu163@163.com.
}

Background In the patients with acute exacerbated chronic obstructive pulmonary disease (AECOPD), the gut displays ischemia, anoxia and oxidative stress, which lead to the intestinal barrier failure. Therefore, it is desirable to screen for effective intestinal barrier-related biomarkers to monitor the disease severity.

Methods: We conducted a prospective observational study in 40 patients with AECOPD and 10 patients with stable chronic obstructive pulmonary disease (COPD). The serum levels of I-FABP, citrulline, D-Lactate, DAO, and $\alpha$-GST, as well as the APACHE II scores were recorded. Person correlation analysis, logistic regression models and receiver operating characteristic (ROC) curve analyses were used in our study.

Results: Patients with AECOPD had significantly higher levels of I-FABP, D-Lactate, and DAO than did those with stable COPD. However, the serum citrulline level was significantly decreased in the patients with stable COPD than in those with AECOPD and the serum $\alpha$-GST was not significantly changed. Additionally, we observed that there was a higher levels of I-FABP, D-Lactate, and DAO and a lower level of citrulline in patients with severe COPD than in patients with nonsevere COPD [APACHE II (nonsevere COPD) <20; APACHE II (severe COPD) $\geq 20$ ]. Correlation analysis showed that I-FABP and D-Lactate had a significantly positive correlations with the APACHE II score, and citrulline had a significantly negative correlations with the APACHE II score. Following, treatment, the levels of I-FABP and D-lactate were decreased and the level of citrulline was increased. Moreover, we screened out the citrulline and DAO, which independently affected the diagnosis of severe COPD by stepwise logistics regression analysis. Additionally, we found that the combination of serum citrulline and DAO can more effectively diagnose the severe COPD than any single biomarker can, which may be a supportive and convenient method that can be used clinically. Conclusions: Serum I-FABP, citrulline and D-Lactate could be used to assess the disease severity. Citrulline and DAO can diagnose the severe COPD and the combination is more effective.

Keywords: Acute exacerbated chronic obstructive pulmonary disease (AECOPD); intestinal barrier; biomarkers; disease severity; diagnostic performance

Submitted Apr 30, 2020. Accepted for publication Sep 28, 2020.

doi: 10.21037/apm-20-1060

View this article at: http://dx.doi.org/10.21037/apm-20-1060 


\section{Introduction}

The characterization of chronic obstructive pulmonary disease (COPD), which involves recurrent inflammation, oxidative stress, protease/antiprotease imbalance, environmental insult, and host genetics, is predominantly associated with abnormal inflammatory processes in the lungs, which leading to irreversible airflow limitation $(1,2)$. Acute exacerbation of chronic obstructive pulmonary disease (AECOPD), as a serious event, is associated with considerably high in-hospital (24\%) and 1-year (59\%) mortality rates (3). Severe episodes of the patients with AECOPD should be admitted to the intensive care unit (ICU) or respiratory intensive care unit (RICU). The Acute Physiology and Chronic Health Evaluation (APACHE II), is a classification system of disease severity, based upon initial values of 12 routine physiologic measurements, age, and previous health status which could provide a general measure of disease severity including AECOPD, and is the most used score in clinical studies to date (4). However, several studies have indicated that the process is complex and tedious due to many variables used in its calculation leading to the consideration of other methods in assessing AECOPD severity $(5,6)$.

In critically ill patients, the intestine is a vulnerable organ, and intestinal barrier dysfunction is common (7). Gastrointestinal disturbance as a potential extrapulmonary systemic consequence of hospitalized AECOPD has been studied in COPD patients $(8,9)$. Some biomarkers in serum had been proposed as markers in monitoring intestinal barrier function $(7,10,11)$. For example, blood intestinal fatty acid-binding protein (I-FABP) is uniquely located at the top of intestinal mucosal villi and is easily to be released into the circulation following enterocyte membrane integrity loss (12). The main source of citrulline in the body is synthesized by enterocytes in the upper part of the villi and the low plasma citrulline concentration is due to the loss of intestinal barrier function in critically ill patients (13). D-Lactate is a product of intestinal bacterial metabolism and is transferred to the portal circulation because of increased intestinal mucosal and capillary permeability (14). Diamine oxidase (DAO) is particularly abundant in enterocytes at the top of small intestinal villi, from where it is released into the peripheral circulation and then inactivated in the liver (15). The $\alpha$-glutathione$S$-transferase $(\alpha-G S T)$ is present in the liver and small intestines and has been suggested to be a sensitive marker of small bowel ischemia (13). Therefore, whether there was a correlation exists between these intestinal barrierrelated biomarkers and the disease severity and whether these intestinal barrier-related biomarkers could be used as a potential biomarkers to predict or assess the disease severity in AECOPD patients remain unclear.

Based on the above issues, we conducted a prospective correlation and diagnosis analyses to further assess the value of intestinal barrier-related biomarkers in predicting or assessing the disease severity in AECOPD patients. We present the following article in accordance with the STROBE Statement reporting checklist (available at http:// dx.doi.org/10.21037/apm-20-1060).

\section{Methods}

\section{Design and patient selection}

This is a prospective observational study conducted by the Department of Respiratory Medicine, Second Affiliated Hospital of the Army Military Medical University (AMMU), China. The study was conducted in accordance with the Declaration of Helsinki (as revised in 2013) and approved by The Army Military Medical University Human Ethics Committee (2018-037-02). Informed consent was taken from all the patients. Based on the formula and actual situation, A total of 40 patients with AECOPD and another 10 with stable COPD were recruited from June 2018 to July 2019 as the AECOPD group and control groups, respectively. The inclusion criteria were a clinical diagnosis of AECOPD according to the Global Initiative for Obstructive Lung Disease (GOLD) guideline 2014 (16); consecutive patients aged between 40 and 90 years, and a BMI of $15-30 \mathrm{~kg} / \mathrm{m}^{2}$; Patients were excluded if they had diseases other than COPD, such as, a history of GI, asthma, lung cancer, or other relevant lung diseases, and a history of severe infection, malignant tumors, and autoimmune diseases.

\section{Sample collection}

Peripheral blood $(5 \mathrm{~mL})$ was sampled from the 40 AECOPD patients and the 10 stable COPD patients within 24 hours of admission by a skilled nurse specialized in collecting blood. The blood samples were sent to the laboratory, immediately and were allowed to stand at room temperature for $1 \mathrm{~h}$, The samples were centrifuged to separate the serum at $1,000 \mathrm{~g}$ for 15 minutes at $4{ }^{\circ} \mathrm{C}$, and then the collected supernatant was further centrifuged at 
Table 1 Tests of normality

\begin{tabular}{lccccccc}
\hline \multirow{2}{*}{$\begin{array}{l}\text { Detection } \\
\text { indicators }\end{array}$} & \multicolumn{3}{c}{ Stable COPD } & & \multicolumn{3}{c}{ AECOPD } \\
\cline { 2 - 4 } \cline { 7 - 8 } & Statistic & df & Sig. & & Statistic & df & Sig. \\
\hline I-FABP & 0.87 & 10 & 0.10 & & 0.98 & 40 & 0.71 \\
Citrulline & 0.99 & 10 & 0.88 & & 0.97 & 40 & 0.33 \\
D-Lactate & 0.85 & 10 & 0.06 & & 0.97 & 40 & 0.32 \\
DAO & 0.95 & 10 & 0.62 & & 0.95 & 40 & 0.08 \\
$\alpha$-GST & 0.97 & 10 & 0.89 & & 0.97 & 40 & 0.43 \\
\hline
\end{tabular}

I-FABP, intestinal fatty acid-binding protein; DAO, diamine oxidase; $\alpha$-GST, $\alpha$-glutathione-S-transferase.

$16,000 \mathrm{~g}$ for 10 minutes at $4^{\circ} \mathrm{C}$. The supernatants were then collected and used for further study. The APACHE II score was assessed by a skilled doctor.

\section{Laboratory analysis}

The human serum I-FABP, citrulline, D-Lactate, DAO, and $\alpha$-GST concentrations were measured using the ELISA kit provided by Sangon Biotech (Shanghai, China) and the results were expressed as $\mathrm{pg} / \mathrm{mL}$ of I-FABP, $\mu \mathrm{mol} / \mathrm{L}$ of citrulline, $\mathrm{mmol} / \mathrm{L}$ of D-Lactate, $\mathrm{mIU} / \mathrm{mL}$ of DAO and $\mathrm{ng} / \mathrm{mL}$ of $\alpha$-GST. The severity of disease was assessed by the APACHE II score. Patients with AECOPD were divided into nonsevere COPD and severe COPD groups according to the APACHE II score (nonsevere COPD $<20$; severe COPD $\geq 20$ ).

\section{Variables}

The serum intestinal barrier-related biomarkers levels were compared between stable COPD patients and AECOPD patients. Patients with AECOPD were divided into nonsevere COPD and severe COPD groups according to the APACHE II score (nonsevere COPD $<20$; severe COPD $\geq 20$ ). The serum intestinal barrier-related biomarkers levels were compared between patients with nonsevere COPD and severe COPD. The correlation was analyzed between the biomarker levels and the APACHE II score. The sensitivity and specificity of the biomarkers for diagnosing the severe COPD were calculated.

\section{Statistical analyses}

Data analyses were performed using SPSS (version 13.0).
As shown in the Table 1, the distribution analysis was performed using tests of normality and the data were in a normal distribution $(\mathrm{P}>0.05)$. Therefore, the data were presented as the means \pm standard deviation. Comparisons were determined by Student's $t$-tests, chi-square tests, ANOVA and paired $t$-tests. Pearson correlation analysis was used to analyze the correlation of biomarkers expression with the APACHE II score. The receiver operating characteristic curve was performed to evaluate the validity of these biomarkers when assessing the disease severity. A P value $<0.05$ was considered to be significant.

\section{Results}

\section{Levels of intestinal barrier-related biomarkers in patients with stable COPD or AECOPD}

To detect the levels of intestinal barrier-related biomarkers, a total of 40 patients with AECOPD and 10 patients with stable COPD were recruited. The baseline characteristics are shown in Table 2. The mean ages of AECOPD, and stable COPD patients were $70.85 \pm 9.13$ and $72.70 \pm 11.13$ years, respectively. Among the AECOPD patients, 33 were men and 7 were women; among the stable COPD patients, 8 were men and 2 were women. The body mass index (BMI) was $20.97 \pm 3.84 \mathrm{~kg} / \mathrm{m}^{2}$ in the AECOPD group and $19.26 \pm 1.77 \mathrm{~kg} / \mathrm{m}^{2}$ in the stable COPD group. Overall, no difference was found between the two groups. Next, the levels of intestinal barrier-related biomarkers were detected by ELISA between patients with stable COPD or AECOPD with no missing data. A significant increase was found in the levels of I-FABP, D-lactate and DAO values in AECOPD patients compared with those in stable COPD patients (Table 3 and Figure 1. However, the values of citrulline were decreased in AECOPD patients compared with those in stable COPD patients (Figure 1). No difference was noted in the $\alpha$-GST value (Figure 1).

\section{Correlation of intestinal barrier-related biomarkers with the severity of $A E C O P D$}

To study the biomarker expression according to the degree of severity in patients with AECOPD, the patients were divided into nonsevere COPD and severe COPD groups according to the APACHE II score (nonsevere COPD $<20$; severe COPD $\geq 20$ ) (17). The I-FABP values were $487.00 \pm 128.60$ and $621.78 \pm 101.38 \mathrm{pg} / \mathrm{mL}$ in the nonsevere COPD and severe COPD, respectively (Table 4). 
Table 2 Baseline characteristics of patients with stable COPD or AECOPD

\begin{tabular}{lccc}
\hline Basic information & Stable COPD $(n=10)$ & Acute exacerbation of COPD $(n=40)$ & $P$ value \\
\hline Age (mean \pm SD, years) & $72.70 \pm 11.13$ & $70.85 \pm 9.13$ & $33: 7$ \\
Sex ratio (man:woman) & $8: 2$ & $20.97 \pm 3.84$ & 0.06 \\
BMI, kg/m ${ }^{2}$ & $19.26 \pm 1.77$ & $16.17 \pm 5.79$ \\
APACHEll & - & 0.18 \\
\hline
\end{tabular}

AECOPD, acute exacerbation of COPD; COPD; chronic obstructive pulmonary disease.

Table 3 Biomarker levels in stable COPD patients and AECOPD patients

\begin{tabular}{lccc}
\hline Detection indicators & Stable COPD $(\mathrm{n}=10)$ & AECOPD $(\mathrm{n}=40)$ & P value \\
\hline I-FABP $(\mathrm{pg} / \mathrm{mL})($ mean $\pm \mathrm{SD})$ & $279.10 \pm 16.29$ & $663.53 \pm 25.25$ & 0.00 \\
Citrulline $(\mathrm{umol} / \mathrm{L})($ mean $\pm \mathrm{SD})$ & $30.54 \pm 1.87$ & $15.14 \pm 0.42$ & 0.00 \\
D-Lactate $(\mathrm{mmol} / \mathrm{L})($ mean $\pm \mathrm{SD})$ & $4.46 \pm 0.27$ & $8.40 \pm 0.27$ & 0.00 \\
DAO $(\mathrm{mlU} / \mathrm{mL})($ mean $\pm \mathrm{SD})$ & $7.14 \pm 0.22$ & $16.17 \pm 0.44$ & 0.00 \\
$\alpha$-GST $(\mathrm{ng} / \mathrm{mL})($ mean $\pm \mathrm{SD})$ & $33.98 \pm 1.61$ & $33.62 \pm 0.72$ & 0.82 \\
\hline
\end{tabular}

AECOPD, acute exacerbated chronic obstructive pulmonary disease; COPD; chronic obstructive pulmonary disease; I-FABP, intestinal fatty acid-binding protein; DAO, diamine oxidase; $\alpha$-GST, $\alpha$-glutathione-S-transferase.

Data analysis showed that the levels of I-FABP were gradually increased with the severity of AECOPD (Figure 2). Similarly, the D-lactate and DAO values were also gradually increased with the severity of AECOPD (Figure 2). However, the citrulline values were $17.86 \pm 1.64 \mu \mathrm{mol} / \mathrm{L}$ and $15.29 \pm 1.62 \mu \mathrm{mol} / \mathrm{L}$ in the nonsevere COPD and severe COPD groups, respectively. Data analysis showed that the levels of citrulline were gradually decreased with the severity of AECOPD (Figure 2).

To compare the relevance between these biomarkers and disease severity, Pearson correlation analysis was used in the current study. A strong association was found between the serum I-FABP level and APACHE II score $(\mathrm{r}=0.62$; $\mathrm{P}<0.05$; Figure 3). Likewise, D-lactate had a strong positive association with the APACHE II score $(\mathrm{r}=0.80 ; \mathrm{P}<0.05$; Figure 3). Additionally, Pearson's correlation of citrulline was -0.89 , which suggested that citrulline had a strong negative correlation with the APACHE II score $(\mathrm{P}<0.05$; Figure 3). However, no correlation was found between DAO and the APACHE II score $(\mathrm{P}>0.05)$ (Figure 3).

\section{The I-FABP and D-Lactate levels are declined and the Citrulline levels are increased following treatment}

Having demonstrated the linkage of the intestinal barrierrelated biomarker levels with the severity of AECOPD, we speculated that the levels of these biomarkers would reduce or increase as the disease resolved following treatment. Accordingly, paired analysis of samples collected before and after treatment demonstrated a reduction in the I-FABP serum levels in 29 of 40 patients after the completion of treatment (Figure 4A). Paired analysis of samples collected before and after treatment demonstrated an increase in the citrulline serum levels in 30 of 40 patients after the completion of treatment (Figure 4B). Reduced serum levels of D-lactate were found in 29 of 40 patients after the completion of treatment (Figure 4C). This result indicated that these indicators improved with the improvement of the disease condition.

\section{Diagnostic properties of plasma biomarkers for patients with severe COPD}

Finally, bivariate logistic regression analysis was used to screen effective warning factors for diagnosing severe COPD. The I-FABP, citrulline, D-lactate, DAO and $\alpha$-GST were treated as independent variables, and the severity of AECOPD was treated as the dependent variable (nonsevere $=0$; severe $=1$ ). From the results of stepwise logistics regression analysis, we screened out citrulline and DAO that independently affected the diagnosis of severe COPD (Table 5). The regression equation was as follows: 
A

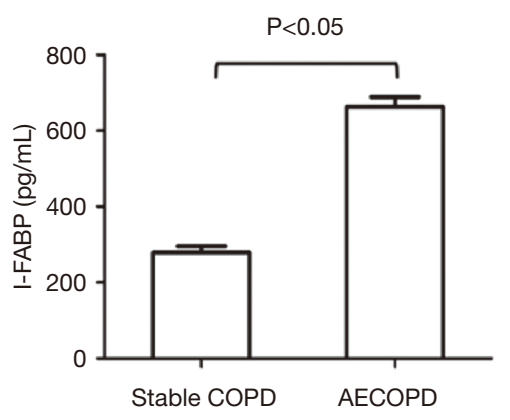

D

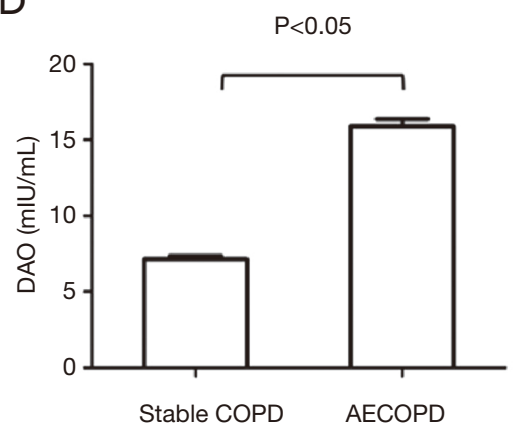

B

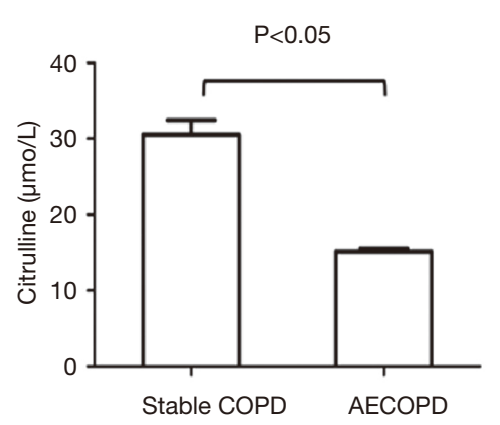

$\mathrm{E}$

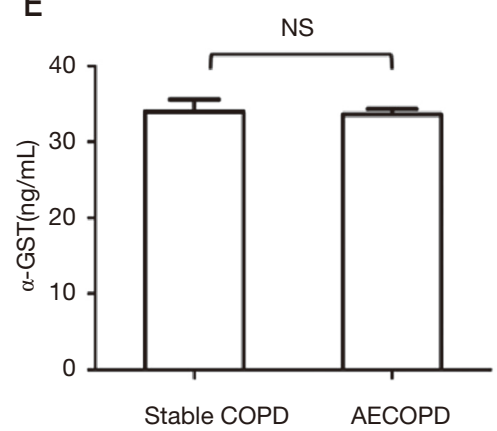

C

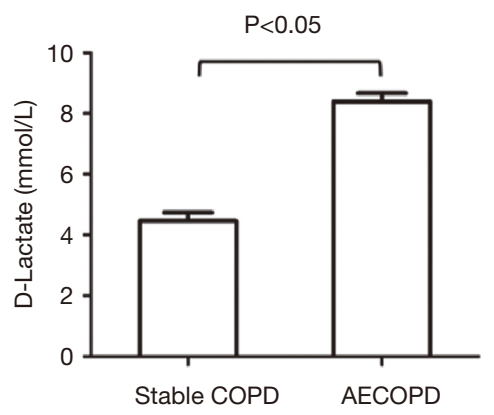

Figure 1 Levels of intestinal barrier-related biomarkers in patients with stable COPD or AECOPD. (A) The concentration of I-FABP was detected by ELISA in AECOPD patients and stable COPD patients, and the I-FABP level was increased in AECOPD patients. (B) The concentration of citrulline was detected by ELISA in AECOPD patients and stable COPD patients, and the citrulline level was decreased in AECOPD patients. (C) The concentration of D-lactate was detected by ELISA in AECOPD patients and stable COPD patients, and the D-lactate level was increased in AECOPD patients. (D) The concentration of DAO was detected by ELISA in AECOPD patients and stable COPD patients, and the DAO level was increased in AECOPD patients. (E) The concentration of $\alpha$-GST was detected by ELISA in AECOPD patients and stable COPD patients, and the $\alpha$-GST level was invariable. AECOPD, acute exacerbated chronic obstructive pulmonary disease; COPD; chronic obstructive pulmonary disease.

Table 4 Biomarker levels in 40 patients with AECOPD

\begin{tabular}{|c|c|c|c|}
\hline Detection indicators & Non-severe group $(\mathrm{N}=30)$ & Severe group $(\mathrm{N}=10)$ & $P$ value \\
\hline I-FABP (pg/mL) (mean \pm SD) & $618.02 \pm 142.65$ & $800.06 \pm 131.67$ & 0.01 \\
\hline Citrulline $(\mu \mathrm{mol} / \mathrm{L})($ mean $\pm \mathrm{SD})$ & $16.06 \pm 1.99$ & $12.40 \pm 2.55$ & 0.00 \\
\hline D-Lactate $(\mathrm{mmol} / \mathrm{L})($ mean $\pm \mathrm{SD})$ & $7.86 \pm 1.42$ & $10.01 \pm 1.50$ & 0.00 \\
\hline
\end{tabular}

AECOPD, acute exacerbated chronic obstructive pulmonary disease; I-FABP, intestinal fatty acid-binding protein; DAO, diamine oxidase.

$y=5.69-1.13 \times 1+0.56 \times 2(\times 1$ represents citrulline; $\times 2$ represents DAO). ROC curve analysis was used to evaluate the diagnostic power of citrulline and DAO (Table 6). ROC curves disclosed that citrulline could diagnose severe COPD (AUC: 0.86), and the curve yielded optimal cut-off values of
$14.28 \mu \mathrm{mol} / \mathrm{L}$ (sensitivity: 0.87; specificity: 0.80) (Figure 5 A). The AUCs were decreased for DAO to diagnose severe COPD (AUC: 0.82) (Figure 5B). The curve-yielded optimal cut-off value was $18.34 \mathrm{mIU} / \mathrm{mL}$ for DAO (sensitivity: 0.60 ; specificity: 0.83 ). Thus, the specificity and sensitivity 
A

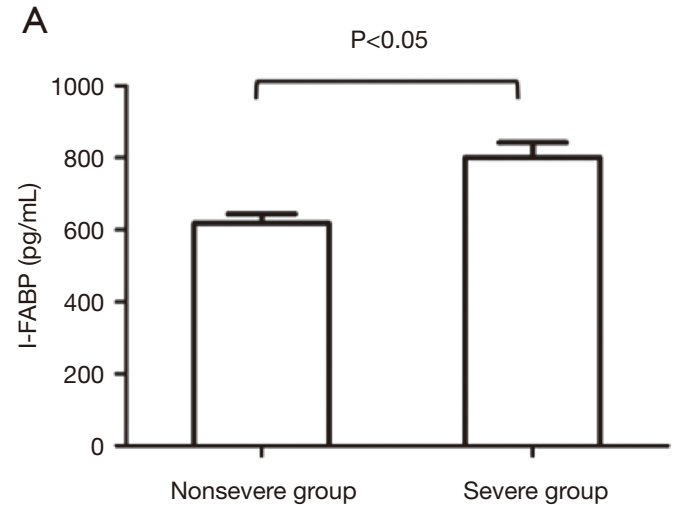

C

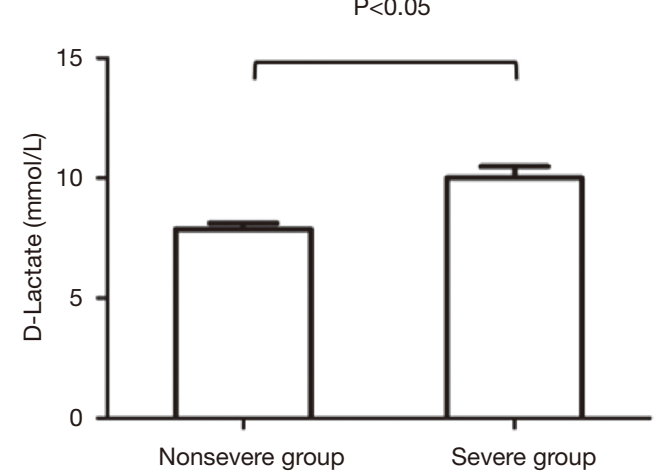

B

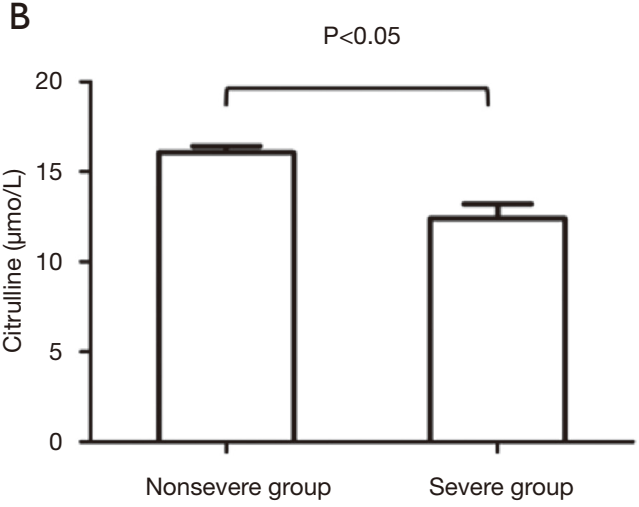

D

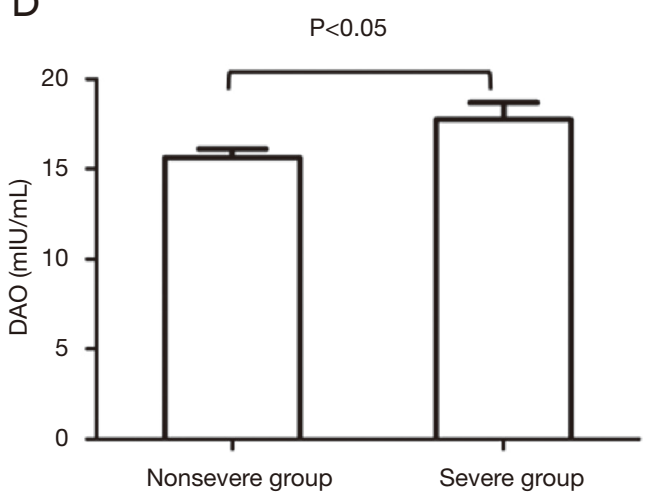

Figure 2 Intestinal barrier-related biomarker expression according to the degrees of severity in patients with AECOPD. (A) Comparison of the concentration of I-FABP in nonsevere COPD and severe COPD. The level of I-FABP was gradually increased. (B) Comparison of the concentration of citrulline in nonsevere COPD and severe COPD. The level of citrulline was gradually decreased. (C) Comparison of the concentration of D-lactate in nonsevere COPD and severe COPD. The level of D-lactate was gradually increased. (D) Comparison of the concentration of DAO in nonsevere COPD and severe COPD. The level of DAO was gradually increased. AECOPD, acute exacerbated chronic obstructive pulmonary disease; COPD; chronic obstructive pulmonary disease.

values of citrulline to diagnose severe COPD were high. The specificity values of DAO were high, but the sensitivity values were low. ROC curve analysis was further conducted, and the AUC value of the combination of citrulline and DAO to diagnose severe COPD was 0.95 . The diagnostic efficacy of the two-marker combination was greater than that of any single biomarker (Figure 5C).

\section{Discussion}

The main highlight of our study was the finding that the levels of I-FABP and D-lactate were significantly increased and that of citrulline was decreased as COPD was exacerbated. Additionally, I-FABP and D-Lactate were strong positive correlated with the disease severity.
Citrulline was strong negative correlated with the disease severity. Following treatment, the levels of I-FABP and D-lactate were decreased and those of citrulline were increased. Moreover, we screened out the citrulline and $\mathrm{DAO}$, which independently affected the diagnosis of severe COPD, and ROC analysis showed that the combination of Citrulline and DAO for effectively diagnosing the severe COPD was more reliable than any single biomarker.

In the patients with AECOPD, gastrointestinal disturbance is a potential extrapulmonary systemic consequence that will result in the translocation of bacteria and release of toxins from the intestinal lumen to the systemic circulation and ultimately aggravating the original disease (18-20). In recent years, several serum biomarkers reflect to intestinal barrier damage have attracted the 
A

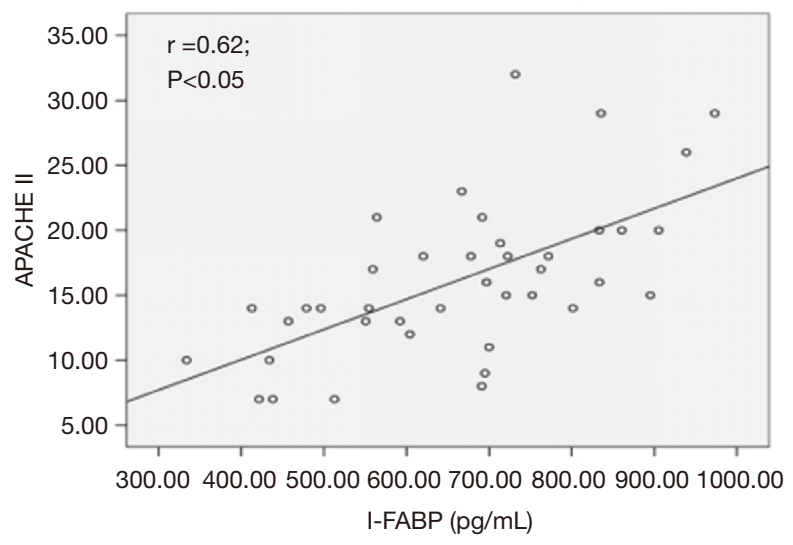

C

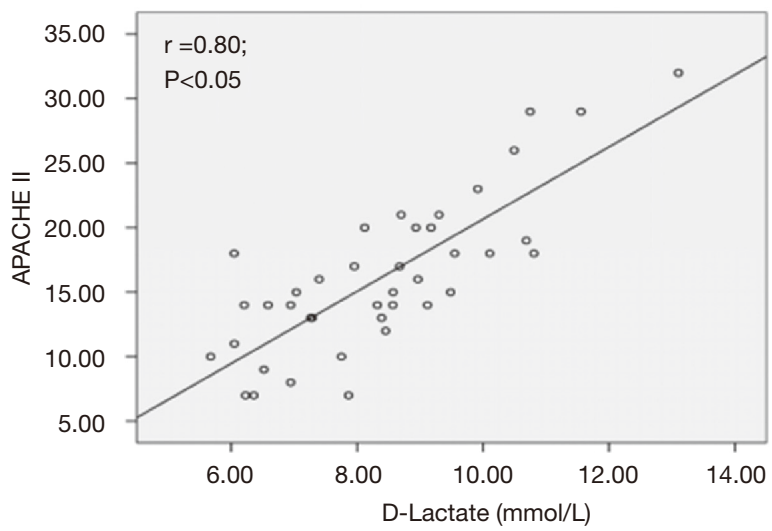

B

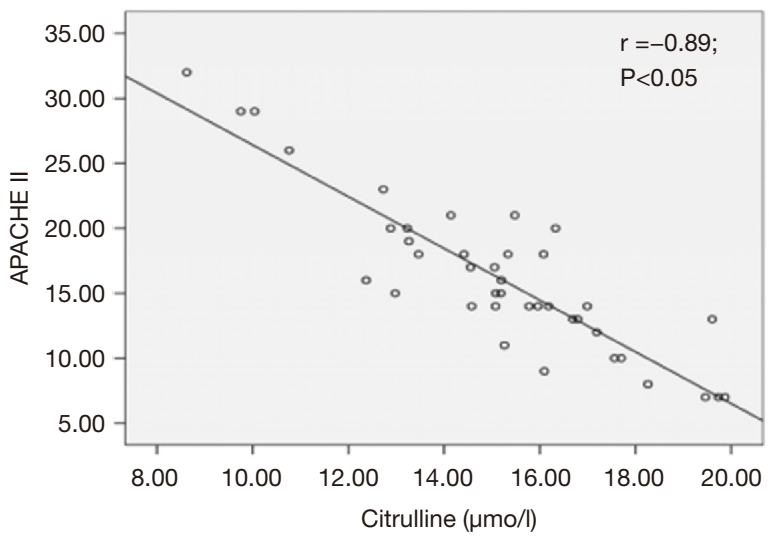

D

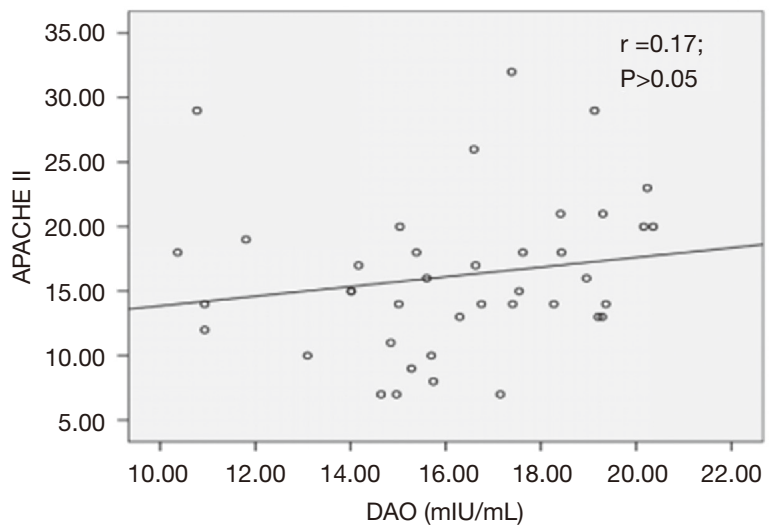

Figure 3 Correlation between the APACHE II score and the level of biomarkers. (A) Correlation between the APACHE II score and I-FABP. There was a strong positive association between serum I-FABP and the APACHE II score. (B) Correlation between the APACHE II score and citrulline. There was a strong negative association between serum citrulline and the APACHE II score. (C) Correlation between the APACHE II score and D-lactate. There was a strong positive association between serum D-lactate and the APACHE II score. (D) Correlation between the APACHE II score and DAO. No association was found between the serum DAO and APACHE II score.

attention of researchers because these biomarkers may react the degree of the patient's condition and predict the risk of the following intestinal barrier dysfunction $(21,22)$. I-FABP, Citrulline, DAO, and $\alpha$-GST are located in enterocytes and are easily to be released into the circulation following the loss of gut barrier function (23). D-lactate is a product of intestinal bacterial metabolism and would transfers to the portal circulation because of increased intestinal mucosal and capillary permeability (14). In the study, we found that patients with AECOPD had significantly higher levels of I-FABP, D-Lactate and DAO and lower levels of citrulline than patients with stable COPD. However, the increase in $\alpha$-GST was not significant. To further research the relevance between these biomarkers and the disease severity, the patients were divided into nonsevere COPD and severe COPD groups according to the APACHE II score (nonsevere COPD $<20$; severe COPD $\geq 20$ ) (17). Our results showed that the I-FABP, D-lactic and DAO values were also gradually increased with the severity of AECOPD. However, the levels of citrulline were gradually decreased with the severity of AECOPD. I-FABP, an intestinal barrier biomarker, has been reported to be associated with the severity of psoriasis, and the relationship may represent a new therapeutic approach for psoriasis (24). Regarding citrulline, the concentration ranged from 20 to $60 \mu \mathrm{mol} / \mathrm{L}$ in healthy adults (25). Additionally, we noticed that the citrulline concentrations were decreased (less than $20 \mu \mathrm{m} / \mathrm{L})$ in critically ill patients $(26,27)$. In Crohn's 
A

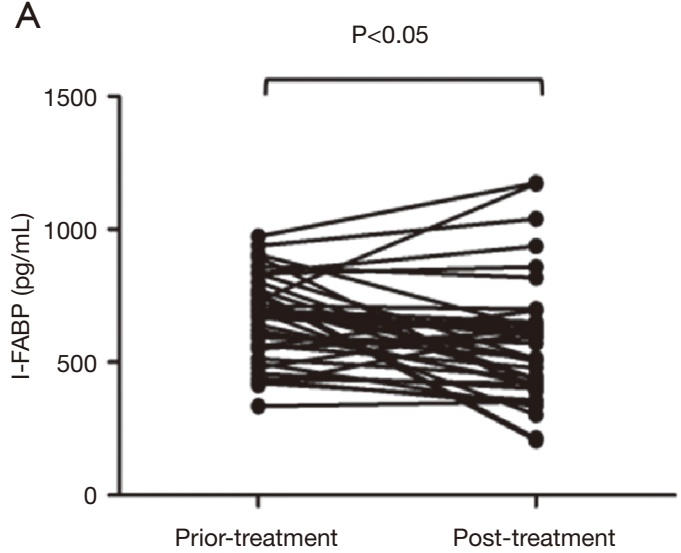

C

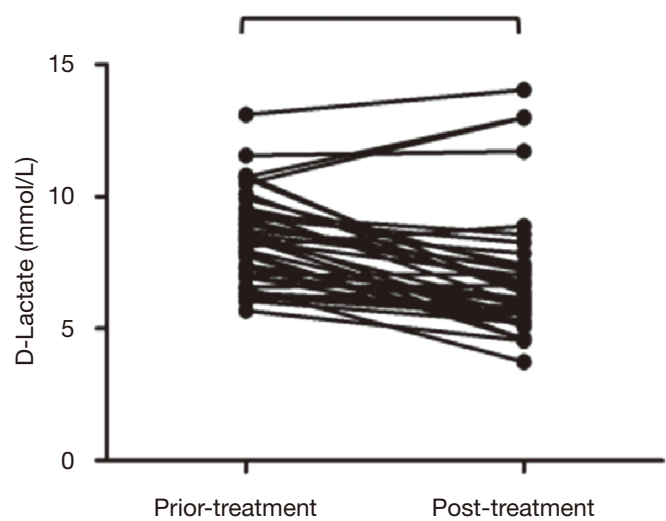

B

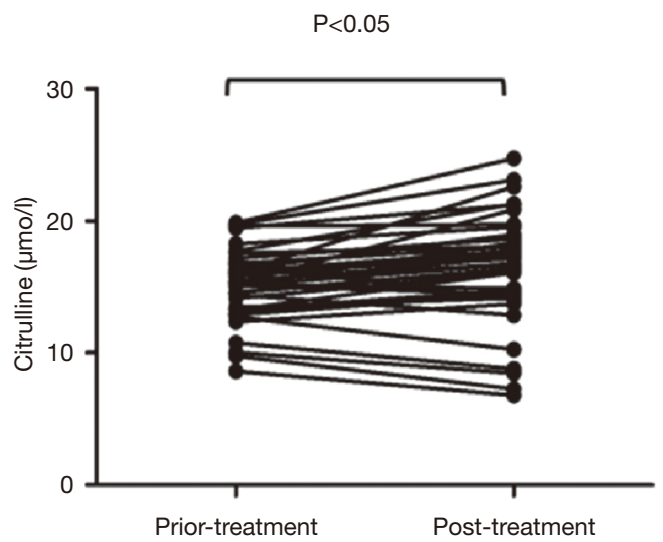

Figure 4 Change in the intestinal barrier-related biomarker levels following treatment. (A) The I-FABP level decreased as the disease resolved following treatment. (B) The citrulline level increased as the disease resolved following treatment. (C) The D-lactate level decreased as the disease was resolved following treatment.

Table 5 Logistic Regression analysis results of the five biomarkers in patients with AECOPD

\begin{tabular}{lcccc}
\hline Projects & B & Sig. & Exp(B) & 95\% Cl for Exp(B) \\
\hline Citrulline $(\mu \mathrm{mol} / \mathrm{L})$ & -1.13 & 0.01 & 0.32 & $0.14-0.73$ \\
DAO $(\mathrm{mlU} / \mathrm{mL})$ & 0.56 & 0.04 & 1.76 & $1.03-2.99$ \\
Constant & 5.70 & 0.22 & 298.61 & \\
\hline
\end{tabular}

AECOPD, acute exacerbated chronic obstructive pulmonary disease; DAO, diamine oxidase.

disease (CD), D-LA and DAO have a good prognostic values for predicting $\mathrm{CD}$ activity (28). In the present study, our findings suggested that monitoring changes in these biomarkers might aid in the evaluation of the disease severity of AECOPD patients.

Pearson's correlation analysis was used to analyze the correlation between the intestinal barrier-related biomarkers and the severity of AECOPD. The results indicated a positive correlation among I-FABP, D-lactate and DAO with the disease severity, but the correlation for DAO was not significant. Additionally, citrulline showed a strong negative correlation with the disease severity. 
Table 6 Diagnostic properties of plasma biomarkers to distinguish severe AECOPD from non-severe AECOPD

\begin{tabular}{|c|c|c|c|c|c|}
\hline Projects & Area under curve & Critical value & Susceptibility & Specificity & $P$ value \\
\hline DAO (mlU/mL) & 0.82 & 18.34 & 0.60 & 0.83 & 0.02 \\
\hline Y & 0.95 & 0.18 & 1 & 0.80 & 0.00 \\
\hline
\end{tabular}

I-FABP, intestinal fatty acid-binding protein; $Y=$ Combination of Citrulline and DAO. AECOPD, acute exacerbated chronic obstructive pulmonary disease; DAO, diamine oxidase.

A

Citrulline

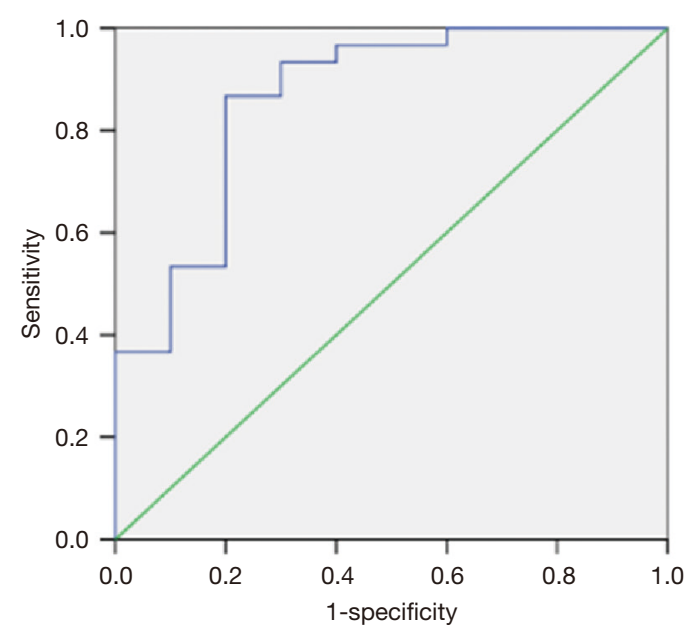

C

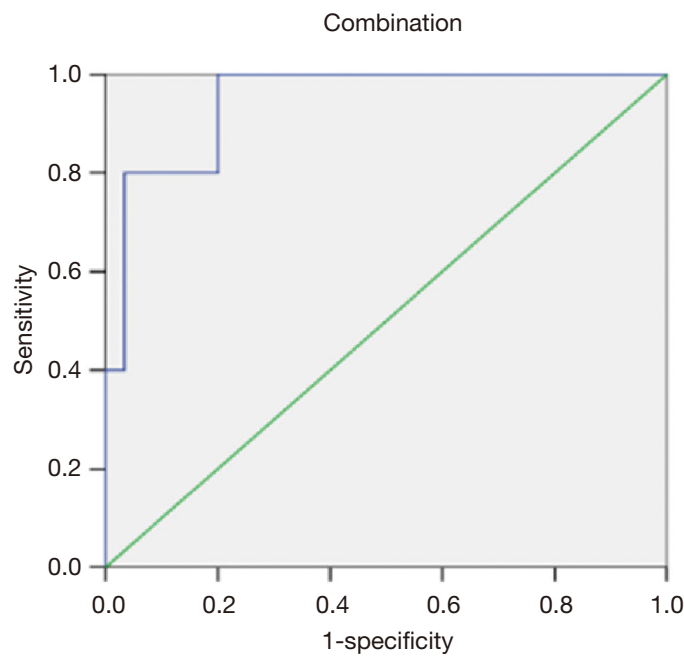

B

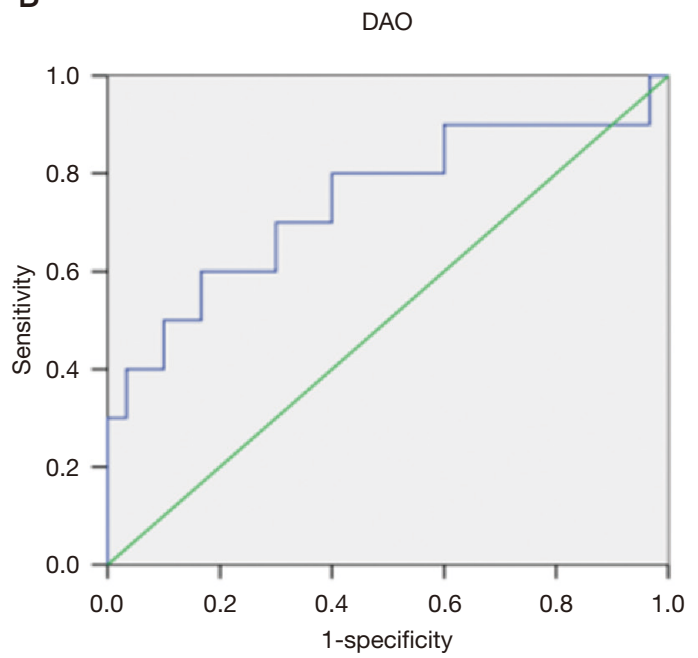

Figure 5 Diagnostic properties of plasma biomarkers for severe COPD. (A) ROC curve analysis revealed that citrulline could diagnose severe COPD. (B) ROC curve analysis revealed that DAO could diagnose severe COPD. (C) ROC curve analysis revealed that the combination of citrulline and DAO could diagnose severe COPD. COPD; chronic obstructive pulmonary disease; ROC, receiver operating characteristic; DAO, diamine oxidase. 
These findings indicated that I-FABP, citrulline and D-lactate could represent the severity of AECOPD well. Furthermore, we observed that I-FABP and D-lactate gradually decreased and citrulline gradually increased as the patient was treated.

Logistic regression analysis was used to screen effective warning factors to diagnose severe COPD. Ultimately, we found that citrulline and DAO could independently affect the diagnosis of severe COPD. To identify the diagnostic value of citrulline and DAO, ROC analysis was performed. In this study, the AUC values of citrulline and DAO were high. The sensitivity and specificity values of citrulline to diagnose severe COPD were high. The sensitivity of DAO was high, but the specificity was low. Finally, we combined the two markers using a logistic regression model to further evaluate the diagnosis efficacy. The AUC value of the combination was higher than any single one and the sensitivity and specificity were also higher, demonstrating that the diagnostic efficacy of the two-biomarkers combination was greater than that of any single biomarker.

\section{Strengths and limitations of the study}

In recent years, the gut-lung axis has been the focus of research. However, most studies have focused on how lung disease affects the intestinal pathological changes. Conversely, we utilized the intestinal barrier-related biomarkers to evaluate the disease severity of AECOPD. In the future, more work on this topic needs to be done. First, we should expand the sample size (because few patients had AECOPD during the study period at our hospital) and will collaborate with other hospitals to increase the number of patients. Additionally, we will expand the variety of diseases. Second, the long-term outcome of the patients with AECOPD should be considered in our study (the research content will be studied in future experiments). Third, more indicators should be included to assess the disease severity rather than only adopting the APACHE II score.

\section{Conclusions}

We found that I-FABP, citrulline and D-lactate were strongly correlated with the disease severity of AECOPD. Additionally, we screened serum intestinal barrier-related biomarkers to predict severe or nonsevere COPD and found that serum citrulline and DAO could be used to diagnose severe COPD. Additionally, the combination of serum citrulline and DAO can more effectively diagnose severe COPD than any single biomarker, which may be a supportive and convenient method to be used clinically.

\section{Acknowledgments}

Funding: This research was supported by grants from the National Natural Science Foundation of China (NSFC81770524 to WX/NSFC81470803 to WX/ NSFC 81700454 to JY); Army Medical University project (2015YLC20 to WX/CX2019JS212 to WX/2019 XQN11 to GD).

\section{Footnote}

Reporting Checklist: The authors have completed the STROBE reporting checklist. Available at http://dx.doi. org/10.21037/apm-20-1060

Data Sharing Statement: Available at http://dx.doi. org/10.21037/apm-20-1060

Peer Review File: Available at http://dx.doi.org/10.21037/ apm-20-1060

Conflicts of Interest: All authors have completed the ICMJE uniform disclosure form (available at http://dx.doi. org/10.21037/apm-20-1060). The authors have no conflicts of interest to declare.

Ethical Statement: The authors are accountable for all aspects of the work in ensuring that questions related to the accuracy or integrity of any part of the work are appropriately investigated and resolved. The study was conducted in accordance with the Declaration of Helsinki (as revised in 2013) and approved by The Army Military Medical University Human Ethics Committee (2018-03702). Informed consent was taken from all the patients.

Open Access Statement: This is an Open Access article distributed in accordance with the Creative Commons Attribution-NonCommercial-NoDerivs 4.0 International License (CC BY-NC-ND 4.0), which permits the noncommercial replication and distribution of the article with the strict proviso that no changes or edits are made and the original work is properly cited (including links to both the formal publication through the relevant DOI and the license). 
See: https://creativecommons.org/licenses/by-nc-nd/4.0/.

\section{References}

1. Brown H, Dodic S, Goh SS, et al. Factors associated with hospital mortality in critically ill patients with exacerbation of COPD. Int J Chron Obstruct Pulmon Dis 2018;13:2361-66.

2. Fischer BM, Voynow JA, Ghio AJ. COPD: balancing oxidants and antioxidants. Int J Chron Obstruct Pulmon Dis 2015;10:261-76.

3. Marchioni A, Castaniere I, Tonelli R, et al. Ultrasoundassessed diaphragmatic impairment is a predictor of outcomes in patients with acute exacerbation of chronic obstructive pulmonary disease undergoing noninvasive ventilation. Crit Care 2018;22:109.

4. Knaus WA, Draper EA, Wagner DP, et al. APACHE II: a severity of disease classification system. Crit Care Med 1985;13:818-29.

5. Naeini AE, Abbasi S, Haghighipour S, et al. Comparing the APACHE II score and IBM-10 score for predicting mortality in patients with ventilator-associated pneumonia. Adv Biomed Res 2015;4:47.

6. Balkan B, Essay P, Subbian V. Evaluating ICU Clinical Severity Scoring Systems and Machine Learning Applications: APACHE IV/IVa Case Study. Annu Int Conf IEEE Eng Med Biol Soc 2018;2018:4073-76.

7. Guo YY, Liu ML, He XD, et al. Functional changes of intestinal mucosal barrier in surgically critical patients. World J Emerg Med 2010;1:205-8.

8. Rutten EP, Lenaerts K, Buurman WA, et al. Disturbed intestinal integrity in patients with COPD: effects of activities of daily living. Chest. 2014;145:245-52.

9. Sprooten RT, Lenaerts K, Braeken D, et al. Increased Small Intestinal Permeability during Severe Acute Exacerbations of COPD. Respiration 2018;95:334-42.

10. Barbeiro HV, Machado M, de Souza HP, et al. Reduction of venous pressure during the resection of liver metastases compromises enteric blood flow: IGFBP-1 as a novel biomarker of intestinal barrier injury. Clinics (Sao Paulo) 2017;72:645-48.

11. Li H, Chen Y, Huo F, et al. Association between acute gastrointestinal injury and biomarkers of intestinal barrier function in critically ill patients. BMC Gastroenterol 2017;17:45.

12. Grootjans J, Thuijls G, Verdam F, et al. Non-invasive assessment of barrier integrity and function of the human gut. World J Gastrointest Surg. 2010;2:61-9.

13. Treskes N, Persoon Am, van Zanten A. Diagnostic accuracy of novel serological biomarkers to detect acute mesenteric ischemia: a systematic review and metaanalysis. Intern Emerg Med 2017;12:821-36.

14. Nielsen C, Kirkegard J, Erlandsen EJ, et al. D-lactate is a valid biomarker of intestinal ischemia induced by abdominal compartment syndrome. J Surg Res 2015;194:400-4.

15. Fukudome I, Kobayashi M, Dabanaka K, et al. Diamine oxidase as a marker of intestinal mucosal injury and the effect of soluble dietary fiber on gastrointestinal tract toxicity after intravenous 5 -fluorouracil treatment in rats. Med Mol Morphol 2014;47:100-7.

16. Asia Pacific COPD Roundtable Group. Global Initiative for Chronic Obstructive Lung Disease strategy for the diagnosis, management and prevention of chronic obstructive pulmonary disease: an Asia-Pacific perspective. Respirology 2005;10:9-17.

17. Hao J, Xu J, Liang Y, et al. Prognostic value of microRNA-122 combined with APACHE II score in patient with acute respiratory distress syndrome. Zhonghua Wei Zhong Bing Ji Jiu Yi Xue. 2019;31:694-8.

18. Vaughan A, Frazer ZA, Hansbro PM, et al. COPD and the gut-lung axis: the therapeutic potential of fibre. J Thorac Dis 2019;11:S2173-80.

19. Tulic MK, Piche T, Verhasselt V. Lung-gut cross-talk: evidence, mechanisms and implications for the mucosal inflammatory diseases. Clin Exp Allergy 2016;46:519-28.

20. Sun Z, Zhu QL, Shen Y, et al. Dynamic changes of gut and lung microorganisms during chronic obstructive pulmonary disease exacerbations. Kaohsiung J Med Sci 2020;36:107-13.

21. Zou L, Song X, Hong L, et al. Intestinal fatty acid-binding protein as a predictor of prognosis in postoperative cardiac surgery patients. Medicine (Baltimore) 2018;97:e11782.

22. Rahman SH, Ammori BJ, Holmfield J, et al. Intestinal hypoperfusion contributes to gut barrier failure in severe acute pancreatitis. J Gastrointest Surg 2003;7:26-36.

23. Kong C, Li SM, Yang H, et al. Screening and combining serum biomarkers to improve their diagnostic performance in the detection of intestinal barrier dysfunction in patients after major abdominal surgery. Ann Transl Med 2019;7:388.

24. Sikora M, Stec A, Chrabaszcz M, et al. Intestinal Fatty Acid Binding Protein, a Biomarker of Intestinal Barrier, is Associated with Severity of Psoriasis. J Clin Med 
2019;8:1021.

25. Crenn P, Messing B, Cynober L. Citrulline as a biomarker of intestinal failure due to enterocyte mass reduction. Clin Nutr 2008;27:328-39.

26. Papadia C, Sherwood RA, Kalantzis C, et al. Plasma citrulline concentration: a reliable marker of small bowel absorptive capacity independent of intestinal inflammation. Am J Gastroenterol 2007;102:1474-82.

Cite this article as: Yin J, Wang S, Qiu Y, Jiang E, Du G, Wang W, Xu P, Yang H, Hu M, Xiao W. Screening for and combining serum intestinal barrier-related biomarkers to predict the disease severity of AECOPD. Ann Palliat Med 2021;10(2):1548-1559. doi: 10.21037/apm-20-1060
27. Tizianello A, De Ferrari G, Garibotto G, et al. Renal metabolism of amino acids and ammonia in subjects with normal renal function and in patients with chronic renal insufficiency. J Clin Invest 1980;65:1162-73.

28. Cai J, Chen H, Weng M, et al. Diagnostic and Clinical Significance of Serum Levels of D-Lactate and Diamine Oxidase in Patients with Crohn's Disease. Gastroenterol Res Pract 2019;2019:8536952. 